\title{
Small-Signal Stability Assessment of Multi-infeed Converter Power System with SVGs
}

This paper was downloaded from TechRxiv (https://www.techrxiv.org).

\section{LICENSE}

CC BY-NC-SA 4.0

SUBMISSION DATE / POSTED DATE

$17-01-2022$ / 20-01-2022

\section{CITATION}

Yuan, Hui; Xin, Huanhai; Wu, Di; Wang, Wei; Zhou, Yuhan (2022): Small-Signal Stability Assessment of Multiinfeed Converter Power System with SVGs. TechRxiv. Preprint. https://doi.org/10.36227/techrxiv.18517862.v1

DOI

10.36227/techrxiv.18517862.v1 


\title{
Small-Signal Stability Assessment of Multi-infeed Converter Power System with SVGs
}

\author{
Hui Yuan, Huanhai Xin, Di Wu, Wei Wang, Yuhan Zhou
}

\begin{abstract}
The modern power gird is being transformed into a multiple converter system (MCS) driven by the increasing integration of converter-based resources (CBRs) such as wind and solar. In such an MCS, the dynamic interaction between converter controls and the power network may cause the small-signal stability issues. While static var generators (SVGs) are integrated into the MCS for providing voltage support, they may affect the small-signal stability. To understand the impact of SVGs, this paper investigates the small-signal stability of the MCS with SVGs. By constructing an equivalent model, the theoretical analysis finds two key factors affecting the small-signal stability when SVGs are connected to the MCS: 1) the topology and parameters of the power network interconnecting SVGs and CBRs; 2) the dynamic interaction between the SVGs and CBRs. Based on the results, a computationally efficient method is proposed to assess the small-signal stability of the MCS with SVGs. The analysis results and the proposed method are validated through eigenvalue analysis, and electromagnetic transient simulation.
\end{abstract}

Index Terms - converter-based resource, multiple converter system (MCS), static var generator (SVG), small signal stability.

\section{INTRODUCTION}

The increasing integration of renewable resources through grid-following converters is shifting the modern power system into a multiple converter system (MCS). In such a MCS, static var generators (SVGs) are widely used for providing voltage support due to their highly flexibility and controllability ${ }^{[1]-[2]}$. However, the integration of SVGs may affect the small-signal stability resulting from the interaction between the converter-based resources (CBRs) and the power network ${ }^{[3]-[8]}$. To support the growing penetration of renewable sources, it is important to understand the impact of SVGs on the small-signal stability of the MCS.

In the literature, different techniques have been used for the small-signal stability analysis resulting from the interaction between CBRs and the power network in grid-connected CBR systems. These techniques can be mainly divided into two categories: eigenvalue analysis based on time-domain model $^{[7],[9]-[10]}$ and the impedance-based analysis in frequency domain $^{[3],[4],[11]-[16]}$. The advantage of eigenvalue analysis is that it can obtain the dominant eigenvalues and the corresponding participation factors of system variables for assessing the small-signal stability and identifying the oscillation sources. It

This work is jointly supported by the National Key R\&D Programme of China (No.2017YFB0902000) and the 2018 Science and Technology Programme of Shaanxi Electric Power Corporation.

H. Yuan, H. Xin, W. Wang and Y. Zhou are with the college of electrical engineering, Zhejiang university, Hangzhou 310027, China (Email: xinhh@zju.edu.cn; Yuan_Hui@zju.edu.cn);

D. Wu is with the department of electrical and computer engineering, North Dakota State University, Fargo, 58102, USA. is challenging to use the eigenvalue analysis for grid-connected CBR systems. It needs to know all system parameters, but the CBRs' inner parameters are not usually provided by the manufacturers with confidentiality concerns. This challenge can be addressed by the impedance (or admittance)-based analysis, which uses the input-output impedance of the CBRs and network to analyze the small signal stability. Since the system impedance can be obtained by frequency scanning without the requirement of CBRs' inner parameters, the impedance-based analysis has been widely used for the small-signal stability analysis of grid-tied CBR systems. Up to now, there are mainly two kinds of impedance-based analysis method for assessing the small-signal stability of grid-tied CBRs: the gain margin and phase margin criterion (GMPMC) ${ }^{[11],[16]}$, and an RLC-circuit-based method provided in [14]. While both of these two methods are based on the reduced one-dimension model by the system impedance matrix, they have differences. The GMPMC uses open-loop gain of system dynamics to quantify the small-signal stability, under the assumption that the reduced one-dimension model has no right-half-plane poles, although this assumption is not always satisfied in practice ${ }^{[15]}$. In [14], authors established an RLC circuit to approximate system dynamics near the resonant frequency and then use it to quantify the small-signal stability. The RLC-circuit-based method may suffer approximate accuracy issues when the system dynamics are complex.

While different techniques have been used in the previous works for the small-signal stability analysis of grid- connected CBRs, these previous works have not analyzed the impact of SVGs on the small-signal stability, especially in the MCS. To fill this gap, this paper investigates the small-signal stability of the MCS with SVGs by constructing an equivalent MCS model. This equivalent model allows us to analytically study how the SVGs affect the small-signal stability. On the basis, a computationally efficient method is proposed for assessing the small-signal stability of the MCS with SVGs. The main contributions of this paper are summarized as follows:

1) An equivalent model of a MCS with SVGs is derived. This equivalent model can consider different control configurations and parameters of CBRs and SVGs. By using the equivalent model, we identify two key factors affecting the small-signal stability of the MCS when integrating SVGs: a) the smallest eigenvalue of weighted network admittance matrix, which are determined by the topology and parameters of the power network as well as the CBR capacity; b) the dynamic interaction between CBRs and SVGs, as characterized by their admittance models.

2) Based on the analysis result, a method is proposed for assessing the small-signal stability of the power system with large-scale integration of CBRs and SVGs. The method is 
computationally efficient since it avoids the requirement for the full-order system model.

3) The analysis results and the proposed method are validated through eigenvalue analysis, and electromagnetic transient simulation with detailed models.

The rest of this paper is organized as follows. Section II introduces the system modelling of the MCS with SVGs. Section III analyzes the small-signal stability of the MCS with SVGs. In Section IV, we present the proposed method for the small-signal stability assessment of the MCS with SVGs. The validation of the analysis results and the proposed method is presented in section V. Section VI draws the conclusions.

\section{MODELING OF MCS WITH SVGs}

In this section, the admittance modelling of a MCS with multiple SVGs is constructed for the small-signal stability analysis. Let us consider a MCS with $k$ SVGs as shown in Fig. 1, where nodes $1 \sim n$ represent CBR nodes; nodes $n+1 \sim n+m$ are passive nodes; SVG1 $\sim$ SVG $k(k \leq m)$ are planned to be integrated at these passive nodes; and the remaining nodes are infinite nodes connected to external grids, which are simplified as ideal voltage sources. In the MCS, the converter can be operated as an CBR or an SVG with the control diagram given in Fig. 2. The control parameters of a CBR or an SVG can be founded in Appendix II. In Fig. 2, $\mathbf{U}_{\mathrm{abc}}^{*}$ and $\mathbf{U}_{\mathrm{abc}}$ are the three-phase internal voltage and terminal voltage of the converter; $\mathbf{I}_{\text {fabc }}$ and $\mathbf{I}_{\text {gabc }}$ are the converter's three-phase current and the grid line's three-phase current; and $Z_{1}$ is the grid line's impedance. The MCS with SVGs can be modelled by integrating the admittance model at the source side (including CBRs and SVGs) with that at the network side.

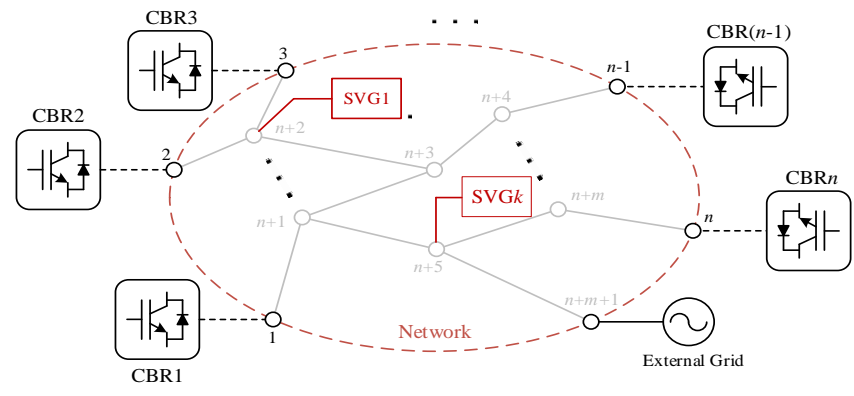

Fig.1 One-line diagram of a typical MCS with SVGs.

\section{A. CBR Modeling}

The admittance model of an CBR can be derived by integrating the modeling of its components including filter inductance and capacitor, current control loop, power control loop, and phase-locked loop.

1) Modeling of Filter inductance and capacitor

The modeling of the filter inductance and the filter capacitor can be derived by using complex transfer functions ${ }^{[17]}$

$$
\begin{aligned}
& . \vec{U}^{*}-\vec{U}=\left(s L_{\mathrm{f}}+j \omega_{\mathrm{pll}} L_{\mathrm{f}}\right) \vec{I} . \\
& \vec{I}_{\mathrm{f}}-\vec{I}_{\mathrm{g}}=\left(s C_{\mathrm{f}}+j \omega_{\mathrm{pll}} C_{\mathrm{f}}\right) \vec{U}
\end{aligned}
$$

where $\vec{U}^{*}=U_{\mathrm{d}}^{*}+j U_{\mathrm{q}}^{*}, \quad \vec{U}=U_{\mathrm{d}}+j U_{\mathrm{q}}, \quad \vec{I}_{\mathrm{f}}=I_{\mathrm{fd}}+j I_{\mathrm{fq}}, \quad$ and $\vec{I}_{\mathrm{g}}=I_{\mathrm{gd}}+j I_{\mathrm{gq}}$ be space vectors of $\mathbf{U}_{\mathrm{abc}}^{*}, \mathbf{U}_{\mathrm{abc}}, \mathbf{I}_{\mathrm{fabc}}$, and $\mathbf{I}_{\mathrm{gabc}}$ in the controller's $d q$-frame, $L_{\mathrm{f}}$ is the filter inductance, $C_{\mathrm{f}}$ is the filter capacitor, $\omega_{\text {pll }}$ is the frequency generated by the phase-locked loop (PLL), and $s$ is the Laplace operator.

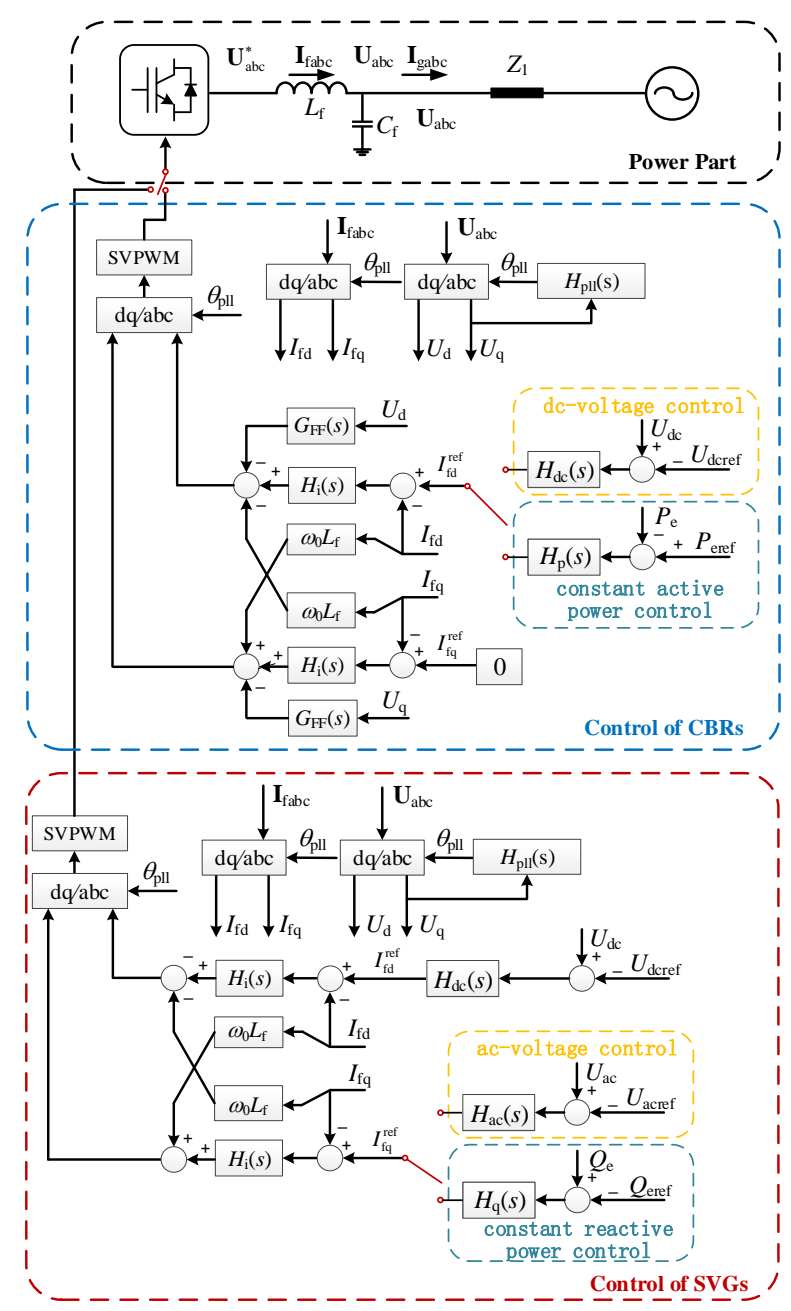

Fig. 2 One-line diagram of a grid-connected converter with a grid-following CBR control or a SVG control.

\section{2) Current control loop}

The dynamic equation of the current control loop is given as

$$
\vec{U}^{*}=H_{\mathrm{i}}(s)\left(\vec{I}_{\mathrm{f}}^{\text {ref }}-\vec{I}_{\mathrm{f}}\right)+j \omega_{\mathrm{pll}} L_{\mathrm{f}} \vec{I}_{\mathrm{f}}+G_{\mathrm{FF}} \vec{U}
$$

where $H_{\mathrm{i}}(s)$ is the transfer function of Proportional-Integral (PI) regulator, $\vec{I}_{\mathrm{f}}^{\text {ref }}=I_{\mathrm{fd}}^{\text {ref }}+j I_{\mathrm{fq}}^{\text {ref }}$ is the current reference vector, and $G_{\mathrm{FF}}(s)$ is the first-order filter of voltage feed-forward control.

\section{3) Modeling of power control loop}

In an CBR, the power control loop includes active and reactive power control loop. To simplify the analysis, the reactive power control loop is omitted, i.e., set $q$-axis current reference $I_{\mathrm{fq}}^{\text {ref }}$ as 0 p.u.. The active power control loop considers two common modes: dc-voltage control and constant active power control ${ }^{[13],[18]}$. The dynamic equation of dc-voltage control loop is given as

$$
\begin{gathered}
I_{\mathrm{fd}}^{\mathrm{ref}}=H_{\mathrm{dc}}(s)\left(U_{\mathrm{dc}}-U_{\mathrm{dcref}}\right) \\
U_{\mathrm{dc}} C_{\mathrm{dc}} s U_{\mathrm{dc}}=P_{\mathrm{m}}-P_{\mathrm{e}} \\
P_{\mathrm{e}}=U_{\mathrm{d}} I_{\mathrm{fd}}+U_{\mathrm{q}} I_{\mathrm{fq}}
\end{gathered}
$$


where $I_{\mathrm{fd}}^{\text {ref }}$ is the d-axis current reference, $H_{\mathrm{dc}}(s)$ is the transfer function of PI regulator, $U_{\mathrm{dc}}$ and $U_{\mathrm{dcref}}$ are respectively the dc voltage and dc voltage reference, $C_{\mathrm{dc}}$ is the dc capacitor, $P_{\mathrm{m}}$ and $P_{\mathrm{e}}$ are respectively the dc capacitor's power input and the converter's active power output, wherein $P_{\mathrm{m}}$ is commonly assumed as a constant value, set as 1 p.u. in this paper.

The dynamic equation of active power control is given as

$$
I_{\mathrm{fd}}^{\text {ref }}=H_{\mathrm{p}}(s)\left(P_{\text {eref }}-P_{\mathrm{e}}\right)
$$

where $H_{\mathrm{p}}(s)$ is the transfer function of PI regulator and $P_{\text {eref }}$ is the active power reference.

4) Modeling of phase-locked loop

The dynamic equation of PLL is given as

$$
\theta_{\mathrm{pll}}=\frac{\omega_{\mathrm{pll}}}{s}=\frac{1}{s} H_{\mathrm{pll}}(s) U_{\mathrm{q}}
$$

where $\theta_{\mathrm{pll}}$ is the PLL's angle output, which is also the angle of the controller's $d q$-frame, $H_{\mathrm{pll}}(s)$ is the transfer function of PI regulator.

In (1)-(8), the components of the output currents and terminals voltage are represented in the $d-q$ local reference frame due to the control blocks in a CBR. To integrate the CBR modeling and the modeling of the power network for the small signal stability analysis of the MCS, the voltage and current vectors in (1)-(8) can be transformed from the controller's $d q$-frame to the global $d q$-frame, wherein the angular frequency is $\omega_{\mathrm{g}}=100 \pi \mathrm{rad} / \mathrm{s}$ and the angle is $\theta_{\mathrm{g}}$, i.e.,

$$
\begin{aligned}
& \vec{I}_{\mathrm{g}}^{\prime}=\vec{I}_{\mathrm{gd}}^{\prime}+j \vec{I}_{\mathrm{gq}}^{\prime}=\vec{I}_{\mathrm{g}} e^{j \delta} \\
& \vec{U}^{\prime}=\vec{U}_{\mathrm{d}}^{\prime}+j \vec{U}_{\mathrm{q}}^{\prime}=\vec{U} e^{j \delta}
\end{aligned}
$$

where $\delta=\theta_{\mathrm{pll}}-\theta_{\mathrm{g}}, \vec{I}_{\mathrm{g}}^{\prime}$ and $\vec{U}^{\prime}$ are the grid line's current and terminal voltage in the global $d q$-frame.

Note that the matrix forms of the above equations based on complex transfer functions are expressed as ${ }^{[17]}$

$$
\begin{aligned}
& y_{\mathrm{d}}+j y_{\mathrm{q}}=\left[G_{\mathrm{d}}(s)+j G_{\mathrm{q}}(s)\right]\left(x_{\mathrm{d}}+j x_{\mathrm{q}}\right) \\
& \Leftrightarrow\left[\begin{array}{l}
y_{\mathrm{d}} \\
y_{\mathrm{q}}
\end{array}\right]=\left[\begin{array}{cc}
G_{\mathrm{d}}(s) & -G_{\mathrm{q}}(s) \\
G_{\mathrm{q}}(s) & G_{\mathrm{d}}(s)
\end{array}\right]\left[\begin{array}{l}
x_{\mathrm{d}} \\
x_{\mathrm{q}}
\end{array}\right]
\end{aligned}
$$

By linearizing (1)-(11), the admittance model of CBRs is given as

$$
\left[\begin{array}{c}
\Delta I_{\mathrm{gd}}^{\prime} \\
\Delta I_{\mathrm{gq}}^{\prime}
\end{array}\right]=-\mathbf{Y}_{\mathrm{CBR}}(s)\left[\begin{array}{c}
\Delta U_{\mathrm{gd}}^{\prime} \\
\Delta U_{\mathrm{gq}}^{\prime}
\end{array}\right]
$$

where $\Delta$ denotes the perturbed value of a variable, $\mathbf{Y}_{\mathrm{CBR}}(s)$ is the 2: 2 admittance matrix of an CBR, whose detailed expression refers to [13]. Note that the admittance matrices of an CBR with the two active power control modes are uniformly represented by $\mathbf{Y}_{\mathrm{CBR}}(s)$ for brevity.

\section{B. SVG Modeling}

Similar to the CBR modeling, the admittance model of an SVG can be derived by integrating the modeling of its components including filter inductance, current control loop, power control loop, and phase-locked loop. However, the modeling of current control loop, and power control loop in the SVG is different from that in CBR. The SVG is commonly operated in zero power factor. That is, the dc capacitor's power input $P_{\mathrm{m}}$ of SVGs is set as 0 p.u., instead of 1 p.u..

\section{1) Current control loop}

In the SVG, the current control loop does not consider the feed-forward voltage control as shown in Fig. $2^{[8]}$, and its dynamic equation is given as

$$
\vec{U}^{*}=H_{\mathrm{i}}(s)\left(\vec{I}_{\mathrm{f}}^{\text {ref }}-\vec{I}_{\mathrm{f}}\right)+j \omega_{\mathrm{pll}} L_{\mathrm{f}} \vec{I}_{\mathrm{f}}
$$

\section{2) Modeling of power control loop}

Two reactive power control modes of SVGs: constant reactive power control and ac voltage control as shown in Fig. 2. The dynamic equation of constant reactive power control loop is given as

$$
\begin{gathered}
I_{\mathrm{fq}}^{\mathrm{ref}}=H_{\mathrm{q}}(s)\left(Q_{\mathrm{e}}-Q_{\text {eref }}\right) \\
Q_{\mathrm{e}}=-U_{\mathrm{d}} I_{\mathrm{fq}}+U_{\mathrm{q}} I_{\mathrm{fd}}
\end{gathered}
$$

where $H_{\mathrm{q}}(s)$ is transfer function of PI regulator, $Q_{\mathrm{eref}}$ and $Q_{\mathrm{e}}$ are reactive power reference and reactive power output of SVGs.

The dynamic equation of ac voltage control loop is given as

$$
I_{\mathrm{fq}}^{\text {ref }}=H_{\mathrm{ac}}(s)\left(U-U_{\text {ref }}\right)
$$

where $H_{\mathrm{ac}}(s)$ is the transfer function of PI regulator, $U_{\text {ref }}$ is the ac voltage reference, set as 1 p.u..

By combining (1), (4)-(6), (8)-(11), (13)-(16), the impedance model of SVGs is derived as

$$
\begin{aligned}
& {\left[\begin{array}{c}
\Delta I_{\mathrm{gd}}^{\prime} \\
\Delta I_{\mathrm{gq}}^{\prime}
\end{array}\right]=-\mathbf{Y}_{\mathrm{SVG}}(s)\left[\begin{array}{c}
\Delta U_{\mathrm{gd}}^{\prime} \\
\Delta U_{\mathrm{gq}}^{\prime}
\end{array}\right]} \\
& \mathbf{Y}_{\mathrm{SVG}}(s)=\left[\begin{array}{ll}
Y_{11}(s) & Y_{12}(s) \\
Y_{21}(s) & Y_{22}(s)
\end{array}\right]
\end{aligned}
$$

where $\mathbf{Y}_{\mathrm{SVG}}(s)$ is the 2! 2 admittance matrix of SVGs, $Y_{11}(s)$, $Y_{12}(s), Y_{21}(s), Y_{22}(s)$ are the elements of $\mathbf{Y}_{\mathrm{SVG}}(s)$.

More specifically, if the SVG is applied with constant reactive power control, $Y_{11}(s), Y_{12}(s), Y_{21}(s), Y_{22}(s)$ can be represented

$$
\left\{\begin{array}{l}
Y_{11}(s)=\frac{C_{\mathrm{dc}} s}{\left(H_{\mathrm{i}}(s)+s L_{\mathrm{f}}\right) C_{\mathrm{dc}} s+U H_{\mathrm{i}}(s) H_{\mathrm{dc}}(s)} \\
Y_{12}(s)=\frac{Y_{12}^{\prime}(s)+I_{\mathrm{fq}} H_{\mathrm{pll}}(s)}{1+U H_{\mathrm{pll}}(s)} \\
Y_{21}(s)=\frac{I_{\mathrm{fq}} H_{\mathrm{i}}(s) H_{\mathrm{q}}(s)}{H_{\mathrm{i}}(s)+s L_{\mathrm{f}}+U H_{\mathrm{i}}(s) H_{\mathrm{q}}(s)} \\
Y_{22}(s)=\frac{1}{\left(1+U H_{\mathrm{pll}}(s)\right)\left(H_{\mathrm{i}}(s)+s L_{\mathrm{f}}+U H_{\mathrm{i}}(s) H_{\mathrm{q}}(s)\right)}
\end{array}\right.
$$

where

$$
Y_{12}^{\prime}(s)=\frac{I_{\mathrm{fq}} H_{\mathrm{i}}(s) H_{\mathrm{dc}}(s)-C_{\mathrm{dc}} L_{\mathrm{f}} I_{\mathrm{fq}} s^{2} H_{\mathrm{pll}}(s)}{\left(H_{\mathrm{i}}(s)+s L_{\mathrm{f}}\right) C_{\mathrm{dc}} s+U H_{\mathrm{i}}(s) H_{\mathrm{dc}}(s)}
$$

If the SVG is applied with ac voltage control, $Y_{11}(s), Y_{12}(s)$, $Y_{21}(s), Y_{22}(s)$ can be represented

$$
\left\{\begin{array}{l}
Y_{11}(s)=\frac{C_{\mathrm{dc}} s}{C_{\mathrm{dc}} s\left(s L_{\mathrm{f}}+H_{\mathrm{i}}(s)\right)+U H_{\mathrm{i}}(s) H_{\mathrm{dc}}(s)} \\
Y_{12}(s)=\frac{Y_{12}^{\prime}(s)+I_{\mathrm{fq}} H_{\mathrm{pll}}(s)}{1+U H_{\mathrm{pll}}(s)} \\
Y_{21}(s)=\frac{-H_{\mathrm{i}}(s) H_{\mathrm{ac}}(s)}{s L_{\mathrm{f}}+H_{\mathrm{i}}(s)} \\
Y_{22}(s)=\frac{1}{\left(1+U H_{\mathrm{pll}}(s)\right)\left(s L_{\mathrm{f}}+H_{\mathrm{i}}(s)\right)}
\end{array}\right.
$$




\section{Power network modeling}

In the MCS shown in Fig. 1, the power network interconnecting all CBRs with SVGs can be modeled by

$$
\Delta \mathbf{I}_{x y}=\boldsymbol{Y}_{\mathrm{N} m}(s) \Delta \mathbf{U}_{x y}
$$

where $\mathbf{I}_{x y} \in \mathbb{R}^{2(n+k) \times 2(n+k)}$ and $\mathbf{U}_{x y} \in \mathbb{R}^{2(n+k) \times 2(n+k)}$ are the vectors representing the current injections and terminal voltages at the CBR nodes and SVG nodes in the global $x y$-frame; $\mathbf{Y}_{\mathrm{N} m}(s)$ is the admittance matrix of the network.

When all the network lines are assumed to have the same proportion $\tau$ of resistance $R$ to inductance $L$ (i.e., $\tau=R / L$ ), $\mathbf{Y}_{\mathrm{N} m}(s)$ can be represented ${ }^{[19]}$

$$
\begin{aligned}
\mathbf{Y}_{\mathrm{N} m}(s)=\mathbf{B}_{\text {red }} \otimes \gamma(s), \gamma(s)=\left[\begin{array}{cc}
\beta(s) & \alpha(s) \\
-\alpha(s) & \beta(s)
\end{array}\right] \\
\mathbf{B}_{\text {red }}=\left[\begin{array}{l|l}
\mathbf{B}_{11} & \mathbf{B}_{12} \\
\hline \mathbf{B}_{21} & \mathbf{B}_{22}
\end{array}\right]=\left[B_{i j}\right]_{(n+k) \times(n+k)} \\
B_{i j}= \begin{cases}-1 /\left(\omega_{0} L_{i j}\right), & i \neq j \\
\sum_{i=1}^{n} 1 /\left(\omega_{0} L_{i j}\right), & i=j\end{cases}
\end{aligned}
$$

where $\otimes$ is the kronecker product, $\mathbf{B}_{\text {red }} \in \mathbb{R}^{(n+k) \times(n+k)}$ is the node-reduced susceptance matrix, wherein only the CBR nodes and SVG nodes are remained, $\mathbf{B}_{11} \in \mathbb{R}^{n \times n}, \mathbf{B}_{12} \in \mathbb{R}^{n \times k}$, $\mathbf{B}_{21} \in \mathbb{R}^{k \times n}, \mathbf{B}_{22} \in \mathbb{R}^{k \times k}$ are the submatrices of $\mathbf{B}_{\text {red }}, B_{i j}$ and $L_{i j}$ are the susceptance and inductance of the line between nodes $i$ and $j, B_{i i}$ is the self susceptance of the $i$ th node, $L_{i i}$ is inductance of the line between node $i$ and infinite nodes (or the ground node), $\alpha(s)=\omega_{0}^{2} /\left[\left(s+\tau \omega_{0}\right)^{2}+\omega_{0}^{2}\right], \beta(s)=\omega_{0}\left(s+\tau \omega_{0}\right) /\left[\left(s+\tau \omega_{0}\right)^{2}+\omega_{0}^{2}\right]$, and $\omega_{0}$ is the synchronous frequency.

\section{Modeling of MCS with SVGs}

To derive the modeling of the MCS as shown in Fig. 1, we first combine the modeling of CBRs in (12) with the modeling of SVGs in (17) to have the integrated admittance model

$$
\Delta \mathbf{I}_{x y}=-\mathbf{Y}_{\mathrm{S} m}(s) \Delta \mathbf{U}_{x y}
$$

where $\mathbf{Y}_{\mathrm{S} m}(s)$ is the admittance matrix at the source side including CBRs and SVGs. More specifically, the admittance matrix $\mathbf{Y}_{\mathrm{S} m}(s)$ can be expressed

$$
\mathbf{Y}_{\mathrm{S} m}(s)=\left[\begin{array}{ll}
\operatorname{diag}\left(S_{\mathrm{B} i} \mathbf{Y}_{\mathrm{CBR} i}(s)\right) & \\
& \operatorname{diag}\left(S_{\mathrm{Bsj}} \mathbf{Y}_{\mathrm{SVGj}}(s)\right)
\end{array}\right]
$$

where $\operatorname{diag}(\cdot)$ denotes a diagonal matrix; $S_{\mathrm{B} i}(i=1, \ldots, n)$ and $S_{\mathrm{Bs} j}$ $(j=1, \ldots, k)$ represent the capacities of CBRs and SVGs, respectively; $\mathbf{Y}_{\mathrm{CBR} i}(s)$ and $\mathbf{Y}_{\mathrm{SVGj}}(s)$ respectively represent the admittance matrices of CBRs and SVGs.

By integrating the source modeling (26) with the power network modeling (22), the MCS with SVGs can be modeled by an equivalent multi-input multi-output feedback control system as shown in Fig. 3 below. The closed-loop characteristic equation of the system can be represented by

$$
\operatorname{det}\left(\mathbf{Y}_{\mathrm{S} m}(s)+\mathbf{Y}_{\mathrm{N} m}(s)\right)=0
$$

where $\operatorname{det}(\cdot)$ denotes the determinant. The system is small-signal stable if and only if all the solutions of (28) (i.e., system eigenvalues) are located in left-half of complex plane.

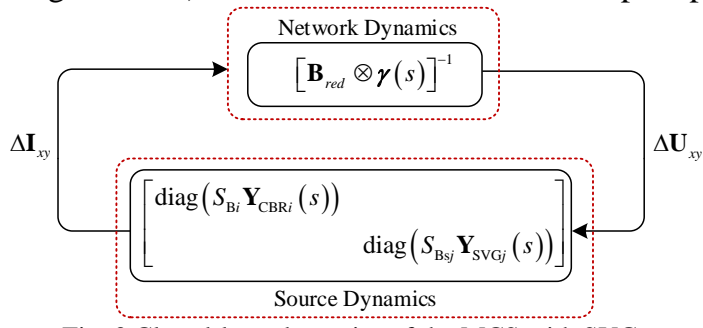

Fig. 3 Closed-loop dynamics of the MCS with SVGs

\section{SMALL-SIGNAL STABILITY ANALYSIS OF MCS WITH SVGS}

While the characteristic equation (28) can be used for the small-signal stability analysis of the MCS with SVGs, such a analysis is challenging because the complex interaction between the CBRs and the power network in the system. Moreover, the analysis complexity is further increased due to the interaction between the CBRs and SVGs interconnected through the power network. Since CBRs and SVGs may have different control configurations or parameters, it is not easy to reduce the analysis complexity based on (28). To address the challenges, we will first construct an equivalent MCS for the small-signal stability analysis. Then, the analysis results are used to reduce the complexity of the small-signal stability analysis of the original MCS with SVGs.

\section{A. Small-signal Stability Analysis of Equivalent MCS}

To this end, the system closed-loop characteristic equation (28) is first modified. When the stability of the MCS with SVGs is mainly caused by the interaction between CBRs and power network, the characteristic equation (28) can be rewritten

$$
\begin{aligned}
& \operatorname{det}\left(\mathbf{Y}_{\mathrm{Sys}}(s)\right)= \\
& \operatorname{det}\left\{\operatorname{diag}\left[\mathbf{Y}_{\mathrm{CBR} i}(s) \gamma^{-1}(s)\right]+\left(\mathbf{S}_{\mathrm{B}}^{-1} \mathbf{B}_{\text {redn }}\right) \otimes \mathbf{I}_{2}+\Delta_{\mathrm{SVG}}(s)\right\} \approx 0 \\
& \mathbf{B}_{\text {redn }}=\mathbf{B}_{11}-\mathbf{B}_{12} \mathbf{B}_{22}^{-1} \mathbf{B}_{21} \\
& \Delta_{\mathrm{SVGs}}(s)=\left[\left(\mathbf{S}_{\mathrm{B}}^{-1} \mathbf{B}_{12} \mathbf{B}_{22}^{-1}\right) \otimes \mathbf{I}_{2}\right] . \\
& \operatorname{diag}\left[S_{\mathrm{Bsj}} \mathbf{Y}_{\mathrm{SVGj}}(s) \gamma^{-1}(s)\right]\left[\left(\mathbf{B}_{22}^{-1} \mathbf{B}_{21}\right) \otimes \mathbf{I}_{2}\right]
\end{aligned}
$$

where

where $\mathbf{Y}_{\text {sys }}(s)$ is the closed-loop transfer function matrix of the MCS with SVGs; $\mathbf{B}_{r e d n}$ is the node-reduced admittance matrix, in which only the CBR nodes remain; $\boldsymbol{S}_{\mathrm{B}}=\operatorname{diag}\left\{S_{\mathrm{B} i}\right\} ; \mathbf{S}_{\mathrm{B}}^{-1} \mathbf{B}_{\text {redn }}$ is the weighted Laplacian matrix; $\Delta_{\mathrm{SVG}}(s)$ is the transfer function matrix related with the dynamics of SVGs and network; $\mathbf{I}_{2}$ is a proper identity matrix. The detailed derivation of (29) is given in Appendix I.

Based on (29), an equivalent MCS can be constructed and its characteristic equation can be represented by

$$
\begin{gathered}
\operatorname{det}\left(\overline{\mathbf{Y}}_{\mathrm{sys}}(s)\right)=\operatorname{det}\left\{\mathbf{I}_{n} \otimes\left[\overline{\mathbf{Y}}_{\mathrm{S}}(s) \gamma^{-1}(s)\right]+\left(\mathbf{S}_{\mathrm{B}}^{-1} \mathbf{B}_{\text {redn }}\right) \otimes \mathbf{I}_{2}\right\}=0 \\
\text { where } \quad \overline{\mathbf{Y}}_{\mathrm{S}}(s)=\sum_{i=1}^{n} p_{1 i} \mathbf{Y}_{\mathrm{CBR} i}(s)+\sum_{j=n+1}^{n+k} p_{2 j} \mathbf{Y}_{\mathrm{SVGj}}(s) \\
p_{1 i}=v_{1 i} u_{1 i}, p_{2 j}=S_{\mathrm{Bs} j} \overline{\mathbf{u}}_{1}^{T}\left[\begin{array}{ll}
\mathbf{S}_{\mathrm{B}}^{-1} & \\
& \mathbf{I}_{k}
\end{array}\right] \mathbf{E}_{s j} \overline{\mathbf{v}}_{1} \\
\overline{\mathbf{u}}_{1}^{T}=\left[\begin{array}{ll}
\mathbf{u}_{1}^{T} & -\mathbf{u}_{1}^{T} \mathbf{S}_{\mathrm{B}}^{-1} \mathbf{B}_{12} \mathbf{B}_{22}^{-1}
\end{array}\right], \overline{\mathbf{v}}_{1}=\left[\begin{array}{c}
\mathbf{v}_{1} \\
-\mathbf{B}_{22}^{-1} \mathbf{B}_{21} \mathbf{v}_{1}
\end{array}\right]
\end{gathered}
$$


where $\overline{\mathbf{Y}}_{\text {sys }}(s)$ is the closed-loop transfer function matrix of the equivalent MCS; $\overline{\mathbf{Y}}_{\mathrm{S}}(s)$ is the dynamic of the source including all CBRs and all SVGs in the equivalent MCS; $\mathbf{E}_{s j}$ is a square matrix, in which only the $j$ th diagonal element is 1 and the other elements are zero; $u_{1 i}$ and $v_{1 i}$ are the $i$ th elements of $\mathbf{u}_{1}^{T}$ and $\mathbf{v}_{1}$; $\mathbf{u}_{1}^{T}$ and $\mathbf{v}_{1}$ are the normalized left eigenvector and right eigenvector for the smallest eigenvalue $\lambda_{1}$ of $\mathbf{S}_{\mathrm{B}}^{-1} \mathbf{B}_{\text {redn }}$.

In the equivalent MCS, its closed-loop transfer function matrix $\overline{\mathbf{Y}}_{\text {sys }}(s)$ (32) can be diagonalized ${ }^{[18]}$

$$
\begin{gathered}
\overline{\mathbf{Y}}_{\mathrm{sys}}(s)=\left[\mathbf{P} \otimes \mathbf{I}_{2}\right] \operatorname{diag}\left[\overline{\mathbf{Y}}_{\mathrm{sysi}}(s)\right]\left[\mathbf{P}^{-1} \otimes \mathbf{I}_{2}\right] \\
\overline{\mathbf{Y}}_{\mathrm{sysi}}(s)=\overline{\mathbf{Y}}_{\mathrm{S}}(s) \boldsymbol{\gamma}^{-1}(s)+\lambda_{i} \mathbf{I}_{2}
\end{gathered}
$$

where $\mathbf{P}$ decomposes $\mathbf{S}_{B}^{-1} \mathbf{B}_{\text {redn }}$ as $\mathbf{P}\left(\mathbf{S}_{B}^{-1} \mathbf{B}_{\text {redn }}\right) \mathbf{P}^{-1}=\operatorname{diag}\left(\lambda_{i}\right)$, $(i=1, \ldots, n)$, in which $\lambda_{i}$ satisfies that $0<\lambda_{1}<\lambda_{2} \leq \cdots \leq \lambda_{n}$; $\overline{\mathbf{Y}}_{\text {syi }}(s)$ is the closed-loop transfer function matrix of an equivalent subsystem with eigenvalue $\lambda_{i}$.

Equation (36) and (37) show that the equivalent MCS can be characterized by $n$ subsystems, and each subsystem has a single equivalent CBR and a single equivalent SVG connected to the infinite node via a line with the equivalent susceptance $B$ equal to eigenvalue $\lambda_{i}$. In each subsystem, the equivalent CBR and the SVG are identical. Thus, the equivalent MCS is stable if and only if all the subsystems are stable.

Further, the small-signal stability of the equivalent MCS can be evaluated based on the smallest eigenvalue $\lambda_{1}$ of matrix $\mathbf{S}_{\mathrm{B}}^{-1} \mathbf{B}_{\text {redn }}$, when the control configuration and parameters of all CBRs and all SVGs are given in the equivalent MCS. Under this condition, the stability of each subsystem depends on $\lambda_{i}$ $(i=1, \ldots, n)$. The smaller $\lambda_{i}$ means its corresponding subsystem is more likely to be unstable $e^{[20]-[21]}$. That is, the small-signal stability of the equivalent MCS depends on the critical subsystem, as shown in Fig. 4, where the smallest eigenvalue $\lambda_{1}$ is viewed as the equivalent line susceptance connecting the equivalent CBR and SVG to the grid. The characteristic equation of the critical subsystem can be represented by (38) below. When the control configuration and parameters of all CBRs and all SVGs are given in the equivalent MCS, the stability of the critical subsystem depends on the smallest eigenvalue $\lambda_{1}$. Thus, the small-signal stability of the equivalent MCS also depends on the smallest eigenvalue $\lambda_{1}$, which is used to evaluate the power grid strength of power systems with renewable energy integration ${ }^{[18]}$.

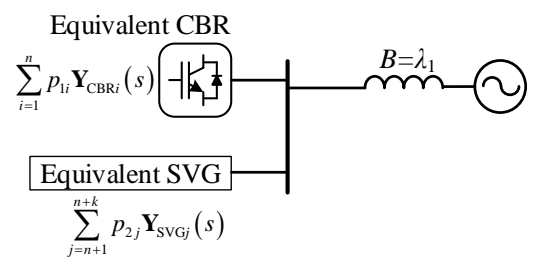

Fig. 4 The critical subsystem decoupled from the equivalent MCS

$$
\operatorname{det}\left(\overline{\mathbf{Y}}_{\mathrm{sys1}}(s)\right)=\operatorname{det}\left(\overline{\mathbf{Y}}_{\mathrm{s}}(s) \gamma^{-1}(s)+\lambda_{1} \mathbf{I}_{2}\right)=0
$$

where $\overline{\mathbf{Y}}_{\mathrm{sys}}(s)$ is the closed-loop transfer matrix of the critical subsystem.
To evaluate the small-signal stability of the equivalent MCS based on the smallest eigenvalue $\lambda_{1}$, let $\lambda_{c}$ be the critical value of $\lambda_{1}$ when the system is critically stable. That is, the $\lambda_{c}$ is the value of $\lambda_{1}$ when the system has a pair of conjugate eigenvalues at the imaginary axis in the complex plane. The $\lambda_{c}$ can be calculated by (39) below. With the $\lambda_{1}$ and its critical value $\lambda_{c}$, we evaluate the small-signal stability and stability margin of the MCS with SVGs. If $\left(\lambda_{1}-\lambda_{c}\right)>0$, the system is stable; otherwise, $\left(\lambda_{1}-\lambda_{c}\right) \leq 0$, the system is unstable. The positive deviation between $\lambda_{1}$ and $\lambda_{c}$ (i.e., $\lambda_{1}-\lambda_{c}$ ) reflects the stability margin of MCSs with SVGs. The larger $\left(\lambda_{1}-\lambda_{c}\right)$ is, the system is more stable.

$$
\operatorname{det}\left(\overline{\mathbf{Y}}_{\mathrm{S}}\left(j \omega_{1}\right) \gamma^{-1}\left(j \omega_{1}\right)+\lambda_{c} \cdot \mathbf{I}_{2}\right)=0
$$

where $\omega_{1}$ is oscillation frequency.

\section{B. Small-signal Stability of the MCS with SVGs}

To use the analysis results from the equivalent MCS to reduce the complexity for the small-signal stability analysis of the original MCS with SVGs, the following Lemma 1 is proposed to prove that the small-signal dynamics of the MCS with SVGs can be characterized by the equivalent MCS.

Lemma 1: Define $y(s)$ and $\bar{y}(s)$ are the dominant eigenvalue functions of the transfer function matrix $\mathbf{Y}_{\mathrm{sys}}(s)$ in (29) and $\overline{\mathbf{Y}}_{\text {sys }}(s)$ in (32), which are pertinent to the system dominant eigenvalues. Then, the loci of $y(s)$ and $\bar{y}(s)$ have the following relationship:

$$
y(s)=\bar{y}(s)+\boldsymbol{o}\left(\left\|\mathbf{Y}_{\text {sys }}(s)-\overline{\mathbf{Y}}_{\text {sys }}(s)\right\|\right)
$$

where $\boldsymbol{o}\left(\left\|\mathbf{Y}_{\text {sys }}(s)-\overline{\mathbf{Y}}_{\text {sys }}(s)\right\|\right)$ denotes the second-order and high-order error.

Proof. Since the stability of the equivalent MCS can be characterized by the critical subsystem as discussed in subsection A in section III, the dominant eigenvalue function $\bar{y}(s)$ of $\overline{\mathbf{Y}}_{\mathrm{sys}}(s)$ is also that of $\overline{\mathbf{Y}}_{\mathrm{sys} 1}(s)$. According to (38),

$\bar{y}(s)$ can be expressed as

$$
\begin{gathered}
\bar{y}(s)=\boldsymbol{\alpha}^{T}(s)\left[\overline{\mathbf{Y}}_{s}(s) \boldsymbol{\gamma}^{-1}(s)+\lambda_{1} \mathbf{I}_{2}\right] \boldsymbol{\beta}(s)=\rho(s)+\lambda_{1} \\
\rho(s)=\boldsymbol{\alpha}^{T}(s)\left[\overline{\mathbf{Y}}_{\mathrm{s}}(s) \boldsymbol{\gamma}^{-1}(s)\right] \boldsymbol{\beta}(s) \\
\boldsymbol{\alpha}(s) \boldsymbol{\beta}(s)=1
\end{gathered}
$$

where $\boldsymbol{\alpha}^{T}(s)$ and $\boldsymbol{\beta}(s)$ are respectively the normalized left eigenvector and right eigenvector of $\overline{\mathbf{Y}}_{\mathrm{S}}(s) \gamma^{-1}(s)$ for the eigenvalue function $\rho(s)$, which is pertinent to the system dominant eigenvalues.

Combining (36) and (41), the dominant eigenvalue function $\bar{y}(s)$ of $\overline{\mathbf{Y}}_{\text {sys }}(s)$ can be written as

$$
\begin{gathered}
\bar{y}(s)=\left[\mathbf{u}_{1}^{T} \otimes \boldsymbol{\alpha}^{T}(s)\right] \overline{\mathbf{Y}}_{\mathrm{sys}}(s)\left[\mathbf{v}_{1} \otimes \boldsymbol{\beta}(s)\right]=\rho(s)+\lambda_{1} \\
{\left[\mathbf{u}_{1}^{T} \otimes \boldsymbol{\alpha}^{T}(s)\right]\left[\mathbf{v}_{1} \otimes \boldsymbol{\beta}(s)\right]=1}
\end{gathered}
$$

where $\mathbf{u}_{1}^{T} \otimes \boldsymbol{\alpha}^{T}(s)$ and $\mathbf{v}_{1} \otimes \boldsymbol{\beta}(s)$ are respectively the normalized left eigenvector and right eigenvector of $\overline{\mathbf{Y}}_{\mathrm{sys}}(s)$ for the dominant eigenvalue function $\bar{y}(s)$. 
Then, consider $\mathbf{Y}_{\text {sys }}(s)$ as a perturbation of $\overline{\mathbf{Y}}_{\text {sys }}(s)$. By the matrix perturbation theory ${ }^{[22]}$, the dominant eigenvalue function $y(s)$ of $\mathbf{Y}_{\text {sys }}(s)$ is written as

$$
\begin{aligned}
y(s) & =\left[\mathbf{u}_{1}^{T} \otimes \boldsymbol{\alpha}^{T}(s)\right] \mathbf{Y}_{\text {sys }}(s)\left[\mathbf{v}_{1} \otimes \boldsymbol{\beta}(s)\right]+\boldsymbol{o}\left(\left\|\mathbf{Y}_{\text {sys }}(s)-\overline{\mathbf{Y}}_{\mathrm{sys}}(s)\right\|\right) \\
& =\bar{y}(s)+\boldsymbol{o}\left(\left\|\mathbf{Y}_{\text {sys }}(s)-\overline{\mathbf{Y}}_{\text {sys }}(s)\right\|\right)
\end{aligned}
$$

This concludes the proof $\mathbf{\square}$

Lemma 1 shows that the dominant eigenvalue function $y(s)$ is approximately equivalent to $\bar{y}(s)$. This indicates that the small-signal stability of the original MCS with SVGs can be characterized by its equivalent MCS. Since the stability of the equivalent MCS is decided by its decoupled critical subsystem, the stability of the orignal MCS with SVGs can also be characterized by the critical subsystem. Further, the stability of the critical subsystem depends on the smallest eigenvalue $\lambda_{1}$ when the control configuration parameters of CBRs and SVGs are given. Thus, the stability of the original MCS with SVGs can be evaluated based on the smallest eigenvalue $\lambda_{1}$. That is, (38) and (39) can be used to analyze the small-signal stability and stability margin of the MCS with SVGs.

Based on (38) and (39), the following significant features about the small-signal stability of the MCS with SVGs can be obtained.

1) The power network plays an important role in the small-signal stability of the MCS with SVGs. It can be observed from (38) that when the control parameters of CBRs and SVGs are given, the system stability depends on the smallest eigenvalue $\lambda_{1}$, which is viewed as the equivalent line susceptance connecting the equivalent CBR and SVG to the grid as shown in Fig. 4. $\lambda_{1}$ is related to network topology and parameters, and the capacities of CBRs. If $\lambda_{1}$ is large, it means that the power network is strong; otherwise, the network is weak. $\lambda_{1}$ is used to evaluate the power grid strength of power systems with renewable energy integrations ${ }^{[18]}$.

2) The interaction between SVGs and CBRs changes the value of $\lambda_{c}$ in (39) to affect the small-signal stability and stability margin of the MCS with SVGs. The interaction is manifested in $\overline{\mathbf{Y}}_{\mathrm{S}}(s)$ in (39). As shown in (33), $\overline{\mathbf{Y}}_{\mathrm{S}}(s)$ includes the dynamics $\mathbf{Y}_{\mathrm{CBR} i}(s)$ of each $\mathrm{CBR}$ and its participation factor $p_{1 i}$ as well as the dynamics $\mathbf{Y}_{\mathrm{SVG}}(s)$ of each SVGs and its participation factor $p_{2 j}$. Thus, the SVGs can affect the interaction between SVGs and CBRs through 1) changing their control strategies and parameters to alter $\left.\mathbf{Y}_{\mathrm{SVG}}(s) ; 2\right)$ changing $p_{2 j}$, which is related to network topology and parameters, SVG locations, and the rated capacities of SVGs and CBRs as shown in (34). Since the sum of $p_{1 i}$ in (34) is a constant value (i.e., $\sum_{i=1}^{n} p_{1 i}=1$ ), if we consider all CBRs as a whole (i.e., $\left.\sum_{i=1}^{n} p_{1 i} \mathbf{Y}_{\mathrm{CBR} i}(s)\right), p_{2 j}(j=n+1, \ldots, n+k)$ can be used to evaluate the degree of the interaction between $\mathrm{SVG} j$ and CBRs through the interconnected power network, when the control parameters of SVGs and CBRs are given. When the larger $p_{2 j}$ is, the stronger the interaction is, or the $\mathrm{SVG} j$ has a larger impact on the $\lambda_{c}$. Specifically, when the SVGs are identical, i.e.,
$\mathbf{Y}_{\mathrm{SVG}(n+1)}(s)=\ldots=\mathbf{Y}_{\mathrm{SVG}(n+k)}(s)$, the dynamics of the equivalent SVG in the critical subsystem can be written as $\mathbf{Y}_{\mathrm{SVG}(n+1)}(s) \sum_{j=n+1}^{n+k} p_{2 j}$. In this case, $\sum_{j=n+1}^{n+k} p_{2 j}$ can be used to evaluate the degree of the interaction between SVGs and CBRs.

\section{Proposed Method for Small Signal Stability ASSESSMENT OF MCSS WITH SVGS}

Based on the analytical results in the previous section, a method is proposed for assessing the small-signal stability of the MCS with SVGs base on the smallest eigenvalue $\lambda_{1}$. This method is illustrated in Fig. 5, and its major steps are summarized:

1) Calculate the smallest eigenvalue $\lambda_{1}$ of matrix $\mathbf{S}_{\mathrm{B}}^{-1} \mathbf{B}_{\text {redn }}$, and acquire the left and right eigenvectors $\left(\boldsymbol{u}_{1}{ }^{\mathrm{T}}\right.$ and $\boldsymbol{v}_{1}$ respectively) corresponding to the smallest eigenvalue $\lambda_{1}$. Then, calculate the participation factor and acquire $p_{1 i}$ in (34) for each converter, and $p_{2 j}$ in (34) for each SVG.

2) Calculate the dynamic model of the equivalent device $\overline{\mathbf{Y}}_{\mathrm{S}}(s)$ in (33), which includes the dynamics of each CBR (i.e., $\mathbf{Y}_{\mathrm{CBR} i}(s)$ ) and SVG (i.e., $\mathbf{Y}_{\mathrm{SVG} j}(s)$ ). We note that the dynamic model of CBRs $\mathbf{Y}_{\mathrm{CBR} i}(s)$ can be obtained by frequency scanning, and the dynamic model of SVGs $\mathbf{Y}_{\mathrm{SVG} j}(s)$ can be calculated with given control parameters.

3) Calculate the critical value $\lambda_{c}$ based on (39). Here, two methods are introduced: 1) the $\lambda_{c}$ can be directly obtained by solving $(39)$; 2) the critical subsystem (38) is first established by experiments; Then, the value of $\lambda_{1}$ is changed in the critical subsystem until the critical subsystem is critically stable by observing the time-domain responses (the corresponding $\lambda_{1}$ is the $\lambda_{c}$ ).

4) Assess the small-signal stability and stability margin by comparing $\lambda_{1}$ with $\lambda_{c}$. If $\lambda_{1}>\lambda_{c}$, then the MCS with SVGs is small-signal stable; the stability margin can be assessed by $\lambda_{1}-\lambda_{c}$; otherwise, if $\lambda_{1} \leq \lambda_{c}$, the MCS with SVGs is small-signal unstable (when the system is critically stable, the system is also considered as unstable).

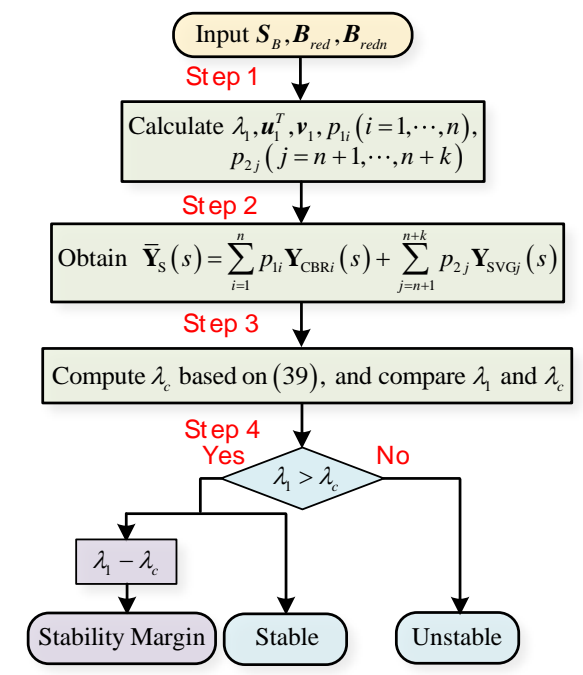

Fig. 5 The process of the practical application process 


\section{V.SIMULATION VALIDATION}

In this section, the analysis results and the proposed method in the previous sections are validated through eigenvalue analysis and electromagnetic transient simulation on a 39-node system as shown in Fig. 6, where node 1 9 are connected to CBRs through a set-up transformer, node 1 3 are connected to SVGs. The capacities of all CBRs are set as 1.2 p.u., and the capacities of all SVGs are set as 1 p.u.. The network parameters can be found in [23], and the parameters of CBRs and SVGs are given in Table I and Table II of the Appendix II.

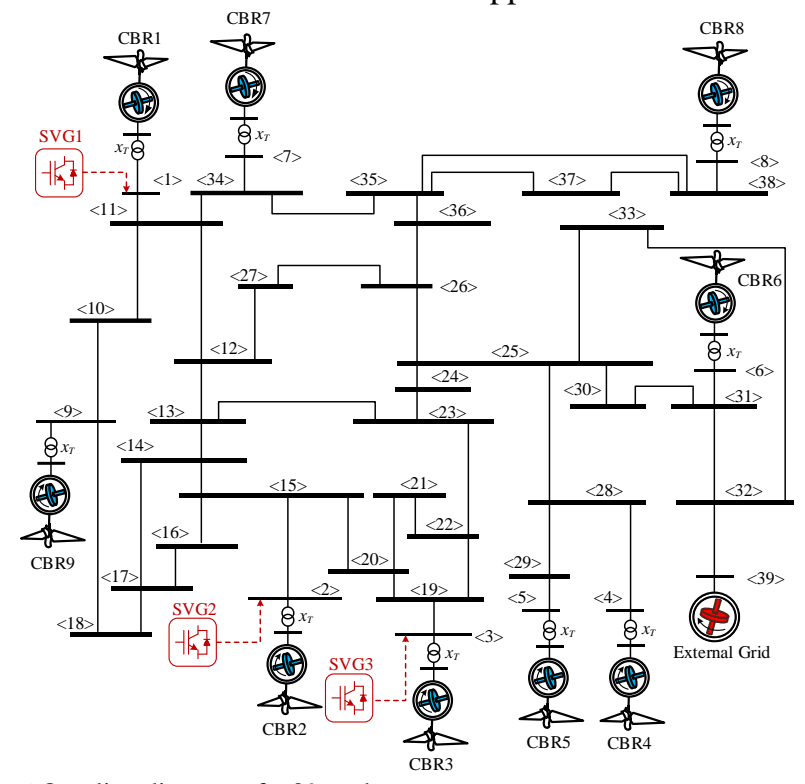

Fig. 6 One-line diagram of a 39-node system

\section{A. Eigenvalue Analysis}

The proposed method for the small-signal stability analysis of the MCS with SVGs is first validated by eigenvalue analysis on the 39-bus system. To this end, in the system all SVGs use the same ac voltage control, but two different scenarios are considered for CBRs: 1) all CBRs use the same dc-voltage control; 2) all CBRs used different controls: CBR1 CBR3 use constant active power control, the other CBRs use dc-voltage control, the PLL's proportional-integral (PI) parameters of CBR1 CBR3 are changed and set up as " 26,7800 ", and the PLL's PI parameters of CBR7 CBR9 are set as "35, 7000". Under the conditions, several cases are created in the system by increasing the rated capacity of CBR1 (i.e., $S_{B 1}$ ) from 0.5 p.u. to 3.0 p.u in scenario 1) (from 0.5 p.u. to 2.5 p.u in scenario 2)) while keeping the capacities of the other CBRs as 1.2 p.u.. Accordingly, we compare the resulting dominant eigenvalues of the 39-node system and the critical subsystem while evaluating the resulting smallest network eigenvalue $\lambda_{1}$, which are shown in Fig. 7.

It can be observed from Fig. 7 that the small-signal stability and stability margin can be evaluated by the smallest eigenvalue $\lambda_{1}$ and its critical value $\lambda_{\mathrm{c}}$. The value $\lambda_{\mathrm{c}}$ can be obtained using (39), and it is 2.478p.u. and 2.694p.u. in the two scenarios when all CBRs have the same strategies or different control strategies. As shown in Fig. 7, when $\lambda_{1}=\lambda_{\mathrm{c}}$, the dominant eigenvalues in the 39-node system is exactly at the imaginary axis, which indicates the system is critically stable. When $\lambda_{1}$ is larger than $\lambda_{c}$, the dominant eigenvalues in the system are in the left-half of the complex plane, which suggests the system is stable and has a certain stability margin. When $\lambda_{1}$ is smaller than $\lambda_{c}$, the dominant eigenvalues in the system are in the right-half of the complex plane, which means the system is unstable. That is, the small-signal stability margin of the system can be identified by $\left(\lambda_{1}-\lambda_{c}\right)$. The larger $\left(\lambda_{1}-\lambda_{c}\right)$ is, more stable the system is. Thus, the efficacy of the proposed method is verified.

Also, it can be observed from Fig. 7 that the small-signal stability of the 39-node system can be characterized by the critical subsystem. As shown in Fig. 7, the dominant eigenvalues of the 39-node system are almost the same as those of the critical subsystem, when all CBRs have either the same control strategies or different control strategies. The observations agree with the analysis results in Section III.
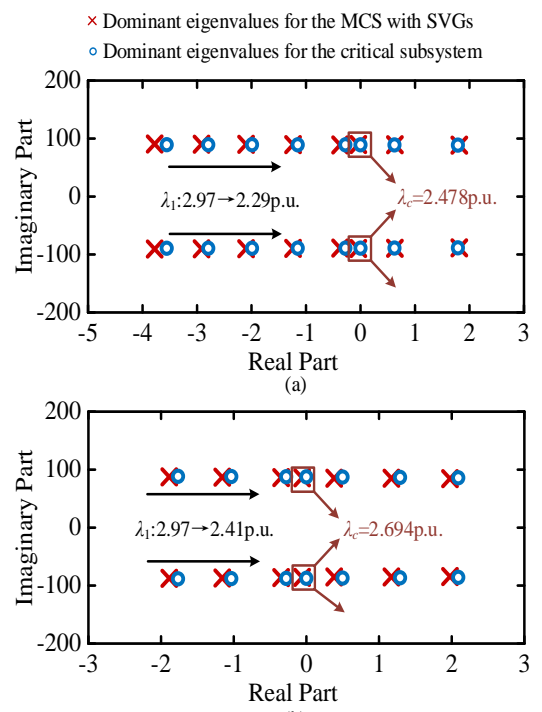

(b)

Fig. 7 The dominant eigenvalues of the 39-node system and the critical subsystem when increasing CBR1's capacity $\left(S_{\mathrm{B} 1}\right)$ and keeping other CBRs' capacities constant under two scenarios: (a) all CBRs in the 39-node system have the same control strategy; (b) all CBRs have different control strategies.

To verify the theoretical analysis in Section III that the dynamics of SVGs can influence the interaction between SVGs and CBRs and thus the system stability (or $\lambda_{c}$ ), let us consider the following four cases, where SVGs use different control strategies or parameters while the capacities of all CBRs are set as 1.2 p.u., and the capacities of all SVGs are set as 1 p.u.. The CBRs use the same dc-voltage control. In the four cases, the resulting $\lambda_{c}$ is calculated based on (39), and the results are shown in Table 1 below. We note that within the elements of $\overline{\mathbf{Y}}_{\mathrm{S}}(s)$ in the critical subsystem (refer to (33)), only $\mathbf{Y}_{\mathrm{SVG}}$, $(j=n+1, \ldots, n+k)$ are changed in the four cases.

Case1): all SVGs use the same ac voltage control, and the proportional parameters $k_{\text {pac }}$ are set as 0.5 ;

Case 2): the case is the same as case 1), but parameters $k_{\text {pac }}$ of all SVGs are increased from 0.5 to 2 ;

Case 3): all SVGs use constant reactive power control, and control parameters refer to Table II of Appendix II;

Case 4): all SVGs are disconnected (as a reference). 
The results of Table 1 verify that various SVG dynamics affect the interaction between SVGs and CBRs through $\overline{\mathbf{Y}}_{\mathrm{s}}(s)$ and thus change the $\lambda_{c}$ in (39) affecting the stability of the 39 -node system. The differences in $\lambda_{c}$ between case 1) and case 2) show the interaction between SVGs and CBRs changes with varying control parameters used in SVGs. The differences in $\lambda_{c}$ between case 1) and case 3) show the interaction changes with varying control strategies used in SVGs. The differences in the $\lambda_{c}$ among cases 1), case 2) and case 4) show the interaction changes when considering the connection of SVGs to the system, and the system may become more stable or less stable with varying control parameters used in SVGs.

Table 1 The critical value $\lambda_{c}$ under four cases

\begin{tabular}{ccccc}
\hline & Case 1) & Case 2) & Case 3) & Case 4) \\
\hline$\lambda_{c}$ & 2.5 & 2.19 & 2.53 & 2.25 \\
\hline
\end{tabular}

In addition to changing the SVG dynamics, changing the participation factor $p_{2 j}$ of SVGs can also affect the interaction between SVGs and CBRs, and thus the system stability as discussed in Section III. In the following four cases, we consider SVGs are integrated in different locations in the system, but all SVGs use the same ac voltage control and all CBRs use the same dc-voltage control. Since the SVGs are identical, the sum of $p_{2 j}$ (i.e., $\sum_{j=n+1}^{n+k} p_{2 j}$ ) can be used to evaluate the degree of the interaction between SVGs and CBRs with given control parameters of SVGs and CBRs, as discussed in subsection B in section III. In the four cases, we evaluate $\sum_{j=n+1}^{n+k} p_{2 j}$ and $\lambda_{c}$, as given in Table 2. We note that within the elements of $\overline{\mathbf{Y}}_{\mathrm{S}}(s)$ in the critical subsystem (refer to (33)), only $p_{2 j},(j=n+1, \ldots, n+k)$ are changed in the four cases.

Case1): three SVGs are connected to the system at node 1 3; Case2): three SVGs are connected to the system at node 3 5; Case3): three SVGs are connected to the system at node 4 6; Case4): three SVGs are disconnected (as a reference).

Table 2 The $\sum_{j=n+1}^{n+k} p_{2 j}$ and $\lambda_{c}$ with varying locations of three SVGs

\begin{tabular}{ccccc}
\hline$/$ & case1) & case2) & case3) & case4) \\
\hline SVGs' location & $1,2,3$ & $3,4,5$ & $4,5,6$ & $/$ \\
$\sum_{j=n+1}^{n+k} p_{2 j}$ & 0.312 & 0.258 & 0.190 & 0 \\
$\lambda_{c}$ & 2.5 & 2.356 & 2.33 & 2.25 \\
\hline
\end{tabular}

The results of Table 2 verify that $\sum_{j=n+1}^{n+k} p_{2 j}$ can be used to evaluate the degree of the interaction between SVGs and CBRs, when the SVGs are identical. The difference of $\lambda_{c}$ among case1, case2, case 3 and case 4 shows that the connections of SVGs increase $\lambda_{c}$ (or deteriorate the system stability). Moreover, the difference of $\lambda_{c}$ among case 1 , case 2 and case 3 shows that the increase of $\sum_{j=n+1}^{n+k} p_{2 j}$ enlarges the impacts of SVGs on $\lambda_{c}$. The simulation results indicate that if $\sum_{j=n+1}^{n+k} p_{2 j}$ is larger under the case that SVGs are identical, the interaction between SVGs and CBRs through power networks are more significant with given control parameters of SVGs and CBRs, which are consistent with the theoretical analysis in Section III.

\section{B. Electromagnetic Transient Simulation}

The proposed method is also validated by electromagnetic transient simulation using MATLAB/Simulink on the 39-node system. Again, in the system all SVGs use the same ac voltage control, but two different scenarios are considered for CBRs: 1) all CBRs use the same dc-voltage control and 2) all CBRs used different controls. The setting of CBR controls in the two scenarios are the same as those used in eigenvalue analysis.

Under the scenario 1), when the rated capacity of CBR1 (i.e., $S_{B 1}$ ) is set as $S_{\mathrm{B} 1}=1.2$ p.u. and $S_{\mathrm{B} 1}=2.4$ p.u., respectively, and the rated capacities of the other CBRs are set as 1.2p.u., the resulting smallest eigenvalue are $\lambda_{1}=2.758$ p.u. and $\lambda_{1}=2.432$ p.u. Under the conditions, a disturbance is applied in the system at node 39 at $t=1 \mathrm{~s}$ to cause 0.05 p.u. of voltage drops and then the voltage is recovered at $t=1.05 \mathrm{~s}$. Fig. 8 shows the time-domain responses of the active power output of all CBRs in the system under the scenario 1) following the disturbance.

Under the scenario 2), when the rated capacity of CBR1 (i.e., $S_{B 1}$ ) is set as $S_{\mathrm{B} 1}=0.5$ p.u. and $S_{\mathrm{B} 1}=1.55$ p.u., respectively, and the rated capacities of the other CBRs are set as 1.2p.u., the resulting smallest eigenvalue are $\lambda_{1}=2.97$ p.u. and $\lambda_{1}=2.66$ p.u.. Under the condition, the same disturbance used in scenario 1) is applied in the system. Fig. 9 shows the time-domain responses of the active power output of all CBRs under the scenario 2) following the same disturbance as that in scenario 1).
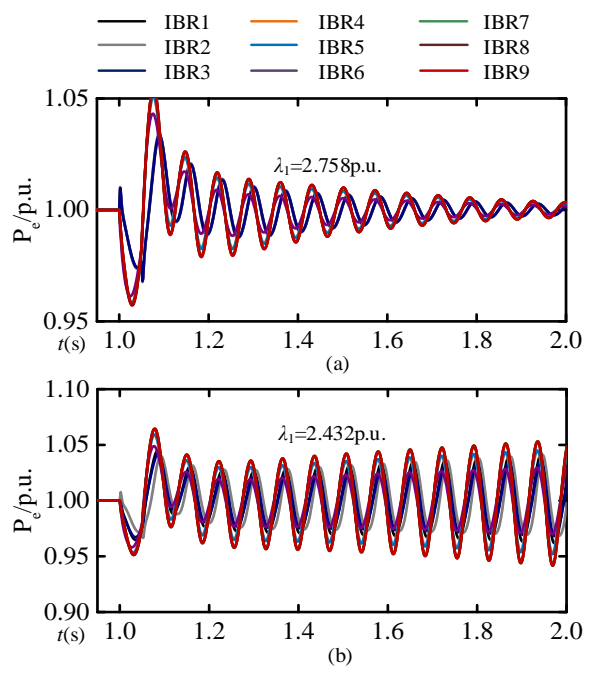

Fig. 8 Time-domain responses of active power outputs of all CBRs with same control strategies in the 39 -node system when $\lambda_{1}=2.758$ p.u. and $\lambda_{1}=2.432$ p.u..

The results of Fig. 8 and Fig. 9 validate the proposed method that the small-signal stability and stability margin of MCSs with SVGs can be evaluated based on the smallest eigenvalue $\lambda_{1}$ and its critical value $\lambda_{c}$. As shown in Fig. 8 and Fig. 9, When $\lambda_{1}=2.758$ p.u. $>\lambda_{\mathrm{c}}=2.478$ p.u. (in Fig. 8) or $\lambda_{1}=2.97$ p.u. $>\lambda_{\mathrm{c}}=$ 2.694p.u. (in Fig.9), the convergent oscillation can be seen, which indicates the original system is stable; When $\lambda_{1}=2.432$ p.u. $<\lambda_{c}=2.478$ p.u. (in Fig. 8) or $\lambda_{1}=2.66$ p.u. $\angle \lambda_{c}=$ 2.694p.u. (in Fig.9), the divergent oscillation can be seen, which means the original system is unstable. The observations 
are consistent with those obtained from Fig. 7 by eigenvalue analysis, which verifies the validity of the proposed method.
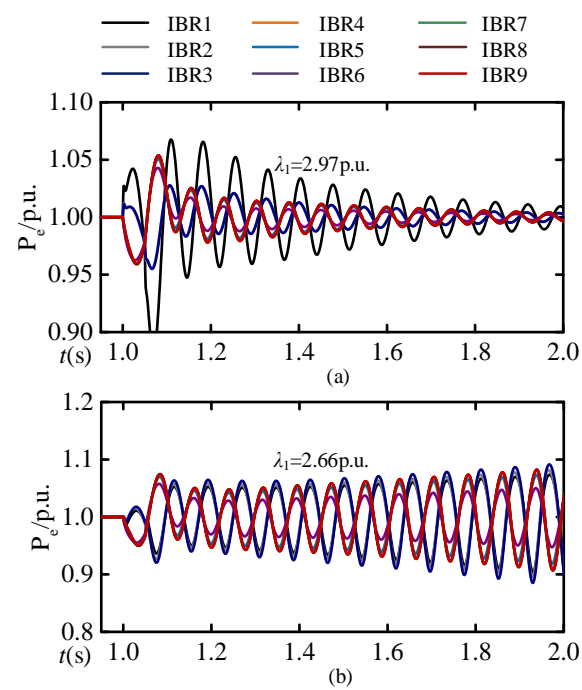

Fig. 9 Time-domain responses of active power outputs of all CBRs with different control strategies in the 39-node system when $\lambda_{1}=2.97$ p.u. and $\lambda_{1}=2.66$ p.u..

\section{CONCLUSIONS}

This paper analyzed the small-signal stability of the MCS with SVGs in order to understand the impact of SVG dynamics on the small-signal stability resulting from the dynamic interaction between CBRs and the power network. To this end, we derived an equivalent MCS model, which can consider the dynamics of CBRs and SVGs with different control configurations and parameters. This equivalent MCS can be decoupled into a set of subsystems, each of which has an identifical equivalent CBR and an identifical equivalent SVG. The small-signal stability of the equivalent MCS depends on the critical subsystem with the smallest eigenvalue of weighted network admittance matrix. It is proved that the small-signal stability of the original MCS with SVGs can be characterized by the equivalent MCS, so the stability of the MCS with SVGs can be analyzed through the critical subsystem. This allows us to gain insights that the small-signal stability of the MCS with SVGs depends on 1) the topology and parameters of power network and 2) the SVG control dynamically interacting with CBRs.

With the insights, a method was proposed for assessing the small-signal stability of the MCS with SVGs. This method does not need the full-order system model, so it reduces computational burden for the small-signal stability analysis of the power system with a large number of CBRs and SVGs. The analysis results and the proposed method were validated through eigenvalue analysis, and electromagnetic transient simulation with detailed models, in which the dynamics of all components in the system are considered without any assumptions and simplifications. The analysis results and proposed method is helpful for guiding grid planning and operation to integrate SVGs into the power systems with high penetration of CBRs.

\section{APPENDIX I}

\section{A. Derivation of (29)}

Based on the closed-loop model in Fig. 3, the closed-loop characteristic equation (28) is detailly expressed as

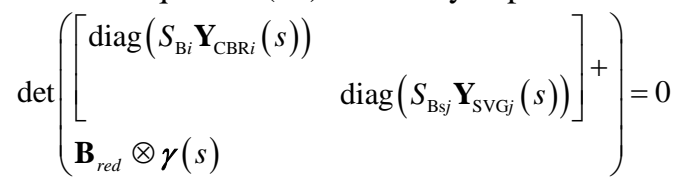

By Schur complement, the characteristic equation (47) is equivalent to

$$
\begin{gathered}
\operatorname{det}\left[\mathbf{C}_{\mathrm{SVGs}}(s)\right] \operatorname{det}\left\{\operatorname{diag}\left[S_{\mathrm{B} i} \mathbf{Y}_{\mathrm{CBR} i}(s) \gamma^{-1}(s)\right]+\mathbf{B}_{11} \otimes \mathbf{I}_{2}-\right. \\
\left.\left[\mathbf{B}_{12} \otimes \mathbf{I}_{2}\right] \mathbf{C}_{\mathrm{SVGs}}^{-1}(s)\left[\mathbf{B}_{21} \otimes \mathbf{I}_{2}\right]\right\} \operatorname{det}\left[\mathbf{I}_{2} \otimes \gamma(s)\right]=0 \\
\mathbf{C}_{\mathrm{SVGs}}(s)=\operatorname{diag}\left[S_{\mathrm{Bsj}} \mathbf{Y}_{\mathrm{SVGj}}(s) \gamma^{-1}(s)\right]+\mathbf{B}_{22} \otimes \mathbf{I}_{2}
\end{gathered}
$$

where $\mathbf{I}_{2}$ is a proper identity matrix; $\operatorname{det}\left[\mathbf{C}_{\mathrm{SVGs}}(s)\right]=0$ in (48) is the characteristic equation of a grid-tied SVGs system, wherein it has $k$ SVGs, and the network parameters are expressed as $\mathbf{B}_{22}$.

With the assumption that the instability of the MCS with SVGs is mainly caused by CBRs (or the SVGs are designed as stable), the closed-loop characteristic equation (48) can be rewritten as

$$
\begin{aligned}
& \operatorname{det}\left\{\operatorname{diag}\left[S_{\mathrm{Bi}} \mathbf{Y}_{\mathrm{CBRi}}(s) \gamma^{-1}(s)\right]+\mathbf{B}_{11} \otimes \mathbf{I}_{2}-\right. \\
& \left.\left[\mathbf{B}_{12} \otimes \gamma(s)\right] \mathbf{C}_{\mathrm{SVGs}}^{-1}(s)\left[\mathbf{B}_{21} \otimes \mathbf{I}_{2}\right]\right\}=0
\end{aligned}
$$

where

$$
\begin{gathered}
\mathbf{C}_{\mathrm{SVGs}}^{-1}(s)=\left\{\mathbf{K}(s)+\mathbf{I}_{2 k}\right\}^{-1}\left[\mathbf{B}_{22}^{-1} \otimes \mathbf{I}_{2}\right] \\
\mathbf{K}(s)=\left[\mathbf{B}_{22}^{-1} \otimes \mathbf{I}_{2}\right] \operatorname{diag}\left[S_{\mathrm{Bsj}} \mathbf{Y}_{\mathrm{SVGj}}(s) \boldsymbol{\gamma}^{-1}(s)\right]
\end{gathered}
$$

where $\mathbf{I}_{k}$ is a proper identity matrix.

It is noteworthy that the spectral radius of $\mathbf{K}(s)$ in our concerned frequency band and damping ratio range (which covers the system dominant eigenvalues) is much less than 1, (i.e., $\rho(\mathbf{K}(s)) \ll 1$, which is presented in Appendix I. B). Then, based on $\rho(\mathbf{K}(s)) \ll 1$ and the Neumann Series ${ }^{[24]}$, the $\left\{\mathbf{K}(s)+\mathbf{I}_{2 k}\right\}^{-1}$ in (51) is approximately expressed as

$$
\left\{\mathbf{K}(s)+\mathbf{I}_{2 k}\right\}^{-1} \approx \mathbf{I}_{2 k}-\mathbf{K}(s)
$$

where the second-order and higher-order of $\mathbf{K}(s)$ is omitted.

Substituting (51)-(53) into (50), the characteristic equation (50) is rewritten as

$$
\begin{aligned}
& \operatorname{det}\left\{\operatorname{diag}\left[S_{\mathrm{Bi}} \mathbf{Y}_{\mathrm{CBRi}}(s) \gamma^{-1}(s)\right]+\left[\left(\mathbf{B}_{12} \mathbf{B}_{22}^{-1}\right) \otimes \mathbf{I}_{2}\right] .\right. \\
& \left.\operatorname{diag}\left[S_{\mathrm{Bsj}} \mathbf{Y}_{\mathrm{SVGj}}(s) \gamma^{-1}(s)\right]\left[\left(\mathbf{B}_{22}^{-1} \mathbf{B}_{21}\right) \otimes \mathbf{I}_{2}\right]+\mathbf{B}_{\text {redn }} \otimes \mathbf{I}_{2}\right\} \approx 0 \\
& \mathbf{B}_{\text {redn }}=\mathbf{B}_{11}-\mathbf{B}_{12} \mathbf{B}_{22}^{-1} \mathbf{B}_{21}
\end{aligned}
$$

Besides, left-multiplying (54) by $\mathbf{S}_{\mathrm{B}}^{-1} \otimes \mathbf{I}_{2},\left(\mathbf{S}_{\mathrm{B}}=\operatorname{diag}\left\{S_{\mathrm{B} 1}, \ldots\right.\right.$, $\left.\left.S_{\mathrm{B} n}\right\}\right)$ obtains that

$$
\operatorname{det}\left\{\operatorname{diag}\left[\mathbf{Y}_{\mathrm{CBRi}}(s) \gamma^{-1}(s)\right]+\left(\mathbf{S}_{\mathrm{B}}^{-1} \mathbf{B}_{\text {redn }}\right) \otimes \mathbf{I}_{2}+\Delta_{\mathrm{SVG}}(s)\right\} \approx 0 \text { (56) }
$$

where

$$
\begin{aligned}
& \Delta_{\mathrm{SVGs}}(s)=\left[\left(\mathbf{S}_{\mathrm{B}}^{-1} \mathbf{B}_{12} \mathbf{B}_{22}^{-1}\right) \otimes \mathbf{I}_{2}\right] . \\
& \operatorname{diag}\left[S_{\mathrm{Bsj}} \mathbf{Y}_{\mathrm{SVGj}}(s) \boldsymbol{\gamma}^{-1}(s)\right]\left[\left(\mathbf{B}_{22}^{-1} \mathbf{B}_{21}\right) \otimes \mathbf{I}_{2}\right]
\end{aligned}
$$




\section{B. Verification of assumption $\rho(\mathbf{K}(s)) \ll 1$}

The rationality of assumption $\rho(\mathbf{K}(s)) \ll 1$ can be verified based on the 39-node system as described in Section V. Let us consider two scenarios: a) the three SVGs use constant reactive controls; b) the three SVGs use ac voltage controls. In the two scenarios, the CBRs use the same dc-voltage control. Fig. 10 shows the loci of $\rho(\mathbf{K}(s))$ under the two scenarios with varying $s$. Here, we define $s=\sigma+j \omega$, wherein $\sigma$ and $\omega=2 \pi f$ are respectively the real part and image part of $s$. We consider the frequency $f$ in $s$ is in range of $[1,100 \mathrm{~Hz}]$, and the damping ratio $\zeta=-\sigma / \sqrt{\sigma^{2}+\omega^{2}}$ in $s$ is in range of $[0,0.1]$. The frequency range and damping ratio range cover the dominant eigenvalues of the MCS related with the sub/super oscillation issues in practice. It can be seen from Fig. 10 that $\rho(\mathbf{K}(s))$ are both smaller than 0.15 for the two scenarios, which are much smaller than 1 . Therefore, the assumption of $\rho(\mathbf{K}(s)) \ll 1$ is rational.
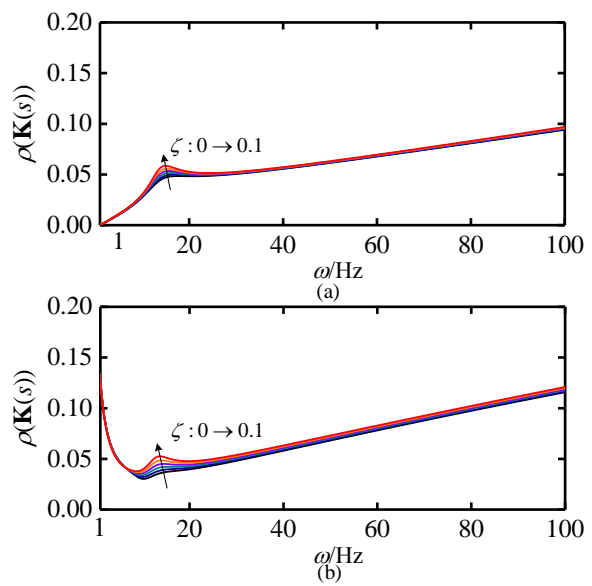

Fig. 10 The loci of $\rho(\mathbf{K}(s))$ with varying $s$ (the frequency in the range [1, $100 \mathrm{~Hz}]$, the damping ratio in the range $[0,0.1]$ ) for two scenarios: (a) the SVG is applied with constant reactive power control; (b) the SVG is applied with ac-voltage control.

\section{APPENDIX II}

Table. I Control parameters of a CBR (per-unit values) Filter inductance $L_{\mathrm{f}}$, filter capacitance $C_{\mathrm{f}}$, dc capacitance $C_{\mathrm{dc}}: 0.05,0.05,0.038$ Transfer function of the current control loop $H_{\mathrm{i}}(s): 1+10 / s$

Transfer function of the dc voltage control loop $H_{\mathrm{dc}}(s): 1+5 / s$

Transfer function of the constant active power control loop $H_{\mathrm{p}}(s): 2+15 / s$

Transfer function of the voltage feedforward filter $G_{\mathrm{FF}}(s): 1 /(1+0.01 s)$

Transfer function of the PLL $H_{\mathrm{pll}}(s):(30+7500 / s) / s$

Parameters of active power output reference and $q$-axis current reference: 1,0

Table. II Control parameters of a SVG (per-unit values) Filter inductance $L_{\mathrm{f}}$, dc capacitance $C_{\mathrm{dc}}: 0.1,0.038$

Transfer function of the current control loop $H_{\mathrm{i}}(s): 1.3+15 / s$

Transfer function of the dc voltage control loop $H_{\mathrm{dc}}(s): 1+5 / s$

Transfer function of the ac voltage control loop $H_{\mathrm{ac}}(s): 0.5+40 / s$

Transfer function of the constant reactive power control loop $H_{\mathrm{q}}(s): 1+5 / s$

Transfer function of the PLL $H_{\mathrm{pll}}(s):(50+7500 / s) / s$

\section{REFERENCES}

[1] X. Wu, M. Wang, M. Shahidehpour, and et. al., "Model-free adaptive control of STATCOM SSO mitigation in DFIG-based wind farm," IEEE Trans. Power Syst., Early ACCESS, DOI: 10.1109/TPWRS.2021. 3082951.
[2] B. Singh, M. Kandpal, and I. Hussain, "Control of grid tied smart PV-DSTATCOM system using an adaptive technique," IEEE Trans, Smart Grid, vol. 9, no. 3, pp. 3986-3993, Sep. 2018.

[3] D. Yang, X. Wang, F. Liu, and et. al., "Symmetrical PLL for SISO impedance modeling and enhanced stability in weak grids," IEEE Trans. Power Electr, vol. 35, no. 2, pp. 1473-1483, 2020.V

[4] X. Xie, Y. Zhan, J. Shair, Z. Ka, and X. Chang, "Identifying the source of subsynchronous control interaction via wide-area monitoring of sub/super-synchronous power flows," IEEE Trans. Power Deliver., vol. 35, no. 5, pp: 2177-2185, 2020.

[5] W. Du, W. Dong, H. Wang, "Small-signal stability limit of a grid-connected PMSG wind farm dominanted by the dynamics of PLLs," IEEE Trans. On Power Syst., vol. 35, no. 3, pp: 2093-2107, 2020.

[6] D. Wang, L. Liang, L. Shi, J. Hu, and et. al., "Analysis of modal resonance between PLL and DC-link voltage control in weak-grid tied VSCs," IEEE Trans. Power Syst. vol. 34, no. 2, pp. 1127-1138, 2019.

[7] L. Fan, "Modeling type-4 wind in weak grids," IEEE Trans. Sustain. Energy, vol. 10, no. 2, 2019.

[8] X. Tian, Y. Chi, Y. Li, and et. al., "Coordinated damping optimization control of sub-synchronous oscillation for DFIG and SVG," CSEE Journal of Power and Energy Systems, vol. 7, no. 1, pp. 140-149, 2021.

[9] Y. Li, L. Fan, and Z. Miao, "Wind in weak grids: Low-frequency oscillations, subsynchronous oscillations, and torsional interactions," IEEE Trans. Power Syst., vol. 35, no. 1, pp. 109-118, 2020.

[10] R. Liu, J. Yao, X. Wang, and et al., "Dynamic stability analysis and improved LVRT schemes of DFIG-based wind turbines during a symmetrical fault in a weak grid," IEEE Trans. Power Electr., vol. 35, no. 1, pp. 303-318, Jan. 2020.

[11] L. Xu, H. Xin, L. Huang, and et. al., "Symmetric admittance modeling for stability analysis of grid-connected converters," IEEE Trans. Energy Conver., vol. 35, no. 1, pp: 434-444, Mar. 2020.

[12] L. Huang, H. Xin, Z. Li, P. Ju, and et al., "Grid-synchronization stability analysis and loop shaping for PLL-based power converters with different reactive power control," IEEE Trans. Smart Grid, vol. 11, no. 1, pp. 501-516, Jan. 2020.

[13] B. Wen, R. Burgos, D. Boroyevich, and et. al., "AC stability analysis and $\mathrm{dq}$ frame impedance specifications in power-electronics-based distributed power systems," IEEE J. Emerg. Selectr. Top. Power Electron., vol. 5, no. 4, pp. 1455-1465, Dec. 2017.

[14] H. Liu, X. Xie, C. Zhang, and et. al., "Quantitative SSR analysis of series-compensated DFIG-based wind farms using aggregated RLC circuit model," IEEE Trans. Power Syst. vol. 32, no. 1, pp. 474-483, Jan. 2017.

[15] Y. Liao, X. Wang, "Impedance-based stability analysis for interconnected converter systems with open loop RHP poles," IEEE Trans. Power Electr., vol. 35, no. 4, pp. 4388-4397, Apr. 2020.

[16] C. M. Wildrick, F. C. Lee, B. H. Cho, and B. Choi, "A method of defining the load impedance specification for a stable distributed power system," IEEE Trans. Power Electron., vol. 10, no. 3, pp. 280-285, May 1995.

[17] L. Harnefors, "Modeling of three-phase dynamic systems using complex transfer functions and transfer matrices," IEEE Trans. Ind. Electron., vol. 54, no. 4, pp. 2239-2248, 2007.

[18] W. Dong, H. Xin, D. Wu, and L. Huang, "Small signal stability analysis of multi-infeed power electronic systems based on grid strength assessment," IEEE Trans. Power syst., vol. 34, no. 2, pp. 1393-1403, 2019.

[19] C. Yang, L. Huang, H. Xin, and et al. "Placing grid-forming converters to enhance small signal stability of PLL-integrated power systems," IEEE Trans. Power syst., 2021, Early access.

[20] J. Zhou, H. Ding, S. Fan, Y. Zhang, and A. Gole, "Impact of short-circuit ratio and phase-locked-loop parameters on the small-signal behavior of a VSC-HVDC converter," IEEE Trans. Power Del., vol. 29, no. 5, pp. 2287-2296, 2014.

[21] Reliability guideline: Integrating inverter-based resources into weak power systems[R]. North American electric reliability corporation, 2017.

[22] G. W. Stewart, Matrix perturbation theory. Citeseer, 1990.

[23] L. Huang, H. Xin, W. Dong, and F. Dörfler, "Impacts of grid structure on PLL-synchronization stability of converter-integrated power systems," arXiv preprint arXiv:1903.05489, 2019.

[24] K. Petersen, M. Pedersen, The matrix cookbook. 2012. 